CLINICAL STUDY

\title{
Mutational analyses of epidermal growth factor receptor and downstream pathways in adrenocortical carcinoma
}

\author{
Ilse G C Hermsen, Harm R Haak, Ronald R de Krijger ${ }^{1}$, Thomas M A Kerkhofs, Richard A Feelders ${ }^{2}$, \\ Wouter W de Herder ${ }^{2}$, Hanneke Wilmink ${ }^{3}$, Jan W A Smit ${ }^{4}$, Hans Gelderblom ${ }^{5}$, Noel F C C de Miranda ${ }^{6}$, \\ Ronald van Eijk ${ }^{6}$, Tom van Wezel $^{6}$ and Hans Morreau ${ }^{6}$ \\ Department of Internal Medicine, Máxima Medical Centre, PO Box 90052, 5600 PD Eindhoven, The Netherlands, Departments of ${ }^{1}$ Pathology and \\ ${ }^{2}$ Endocrinology, Erasmus MC-University Medical Center, Rotterdam, The Netherlands, ${ }^{3}$ Department of Medical Oncology, Amsterdam Medical Centre, \\ Amsterdam, The Netherlands, Departments of ${ }^{4}$ Endocrinology, ${ }^{5}$ Clinical Oncology and ${ }^{6}$ Department of Pathology, Leiden University Medical Centre, \\ Leiden, The Netherlands
}

(Correspondence should be addressed to I G C Hermsen; Email: ilsehermsen@gmail.com)

\begin{abstract}
Background: Adrenocortical carcinoma (ACC) is a rare disease with a poor prognosis and limited therapeutic options. Mitotane is considered the standard first-line therapy with only $30 \%$ of the patients showing objective tumour response. Defining predictive factors for response is therefore of clinical importance.

The epidermal growth factor receptor (EGFR) has been implicated in the development of one-third of all malignancies. EGFR pathway members in ACC have been investigated, however, without available clinical data and relation to survival.

Methods: In this study, mutation status of EGFR and downstream signalling pathways was evaluated in 47 ACC patients on mitotane using direct sequencing, a TaqMan allele-specific assay and immunohistochemistry. Archival formalin-fixed paraffin-embedded tumour tissue was used for all analyses. Patient data were obtained anonymously, after coupling with the collected tumour tissue. Results: One BRAF, two EGFR TK domain (c.2590G > A, p.864A > T) and 11 TP53, but no PIK3CA or $K R A S$, mutations were found. No relationship was found between mutation status, immunostaining and mitotane response or survival.

Conclusion: In conclusion, our data suggest that the role of EGFR tyrosine kinase inhibitors in ACC is limited. Treatment with EGFR monoclonal antibodies on the other hand might be beneficial for a larger group of patients. The possible efficacy of this therapy in ACC should be evaluated in future trials.
\end{abstract}

European Journal of Endocrinology 169 51-58

\section{Introduction}

Adrenocortical carcinoma (ACC) is considered one of the most aggressive solid tumours with a poor prognosis. Fifty years after its first use, mitotane (o,p'-DDD) is still considered the most effective drug for ACC. Nonetheless, a number of limitations for this treatment exist including poor bioavailability, side effects and a narrow therapeutic range. Furthermore, the mechanism of action is not well known (1). Although mitotane treatment has its limitations, response to mitotane monotherapy has been associated with a better prognosis $(2,3,4)$. As only $30 \%$ of patients on mitotane monotherapy show objective tumour response $(3,4,5)$, defining these patients would be of clinical importance.

Until now, poor understanding of ACC pathogenesis has hampered the development of new therapies. Gene expression profiling has been successfully used to improve this understanding. With this technique, subgroups of ACC with different gene expression profiles and clinical outcomes have been identified $(6,7)$. P53 and $\beta$-catenin, for example, were shown to be poor prognostic factors in ACC (8). In addition to molecular profiling, the search for new therapies has resulted in the investigation of several potential candidates (9).

Targeted tailor-made therapy for individual patients has been applied in advanced stages of several cancers (10). Most of these treatments are directed towards molecular pathways in the tumour cells that are constitutively activated in these cancers (based on the so-called oncogene addiction theory). An important therapy target is the epidermal growth factor receptor (EGFR) pathway. EGFR is involved in the regulation of many cell processes such as cell growth, proliferation and apoptosis, and it has been implicated in the development of $30 \%$ of all cancers (11). As a result, two anti-EGFR therapies have been developed, tyrosine kinase inhibitors (TKIs) and monoclonal antibodies (MABs), both potential new therapies in ACC. EGFR pathway involvement in ACC has been investigated in a 
few studies; however, only limited clinical data are available while the relationship with survival is unknown.

In order to improve prediction of tumour response to mitotane treatment and further study the involvement of EGFR pathway in the tumour genesis of ACC, we determined the mutation status of EGFR and downstream effector molecules, stratifying also for TP53 mutation status as well as for $\beta$-catenin expression in a Dutch cohort of ACC patients treated with mitotane monotherapy.

\section{Materials and methods}

\section{Tumour specimens and clinical data}

Archival formalin-fixed paraffin-embedded tumour tissue (FFPE) of 47 ACC patients treated with mitotane monotherapy, in four Dutch hospitals, i.e. Leiden University Medical Centre, Erasmus MC - University Medical Centre Rotterdam, Amsterdam Medical Centre, Amsterdam, and Maxima Medical Centre Eindhoven, was collected. Clinical data were obtained from each patient. Follow-up was available for all patients from date of diagnosis until January 2010. After collection of the tumour tissue blocks, histopathological slides of each case were reviewed by two pathological experts in adrenal disease. In all cases, the diagnosis of ACC was confirmed as all samples had van Slooten scores $>8$ (12) and Weiss scores $>3$ (13). Furthermore, malignant nature was confirmed by the presence of stage IV disease or recurrent disease in all patients. After coupling of the clinical data to the pathology specimen, both patient information and tumour tissue were anonymised.

\section{Definitions}

Mitotane monotherapy was defined as treatment with mitotane (Lysodren, HRA Pharma, Paris, France) tablets as single treatment. Patients with concomitant chemotherapy or radiation therapy were excluded from the study. Response to mitotane treatment was assessed by CT-examination, at least every 2-3 months. Evaluation of tumour response was based on the RECIST criteria version 1.0 (14). Responders were defined as patients with radiological partial (PR) or complete (CR) tumour response to mitotane therapy. Non-responders were defined as patients with progressive disease during mitotane treatment. Staging was performed using the ENS@T staging criteria (15). According to ENS@T staging, the stages are defined as follows: stage I, tumour size $<5 \mathrm{~cm}$ without any risk factor (T1NOM0); stage II, tumour size $>5 \mathrm{~cm}$ but still without risk factors (T2NOMO); stage III, tumour of any size with at least one of the following factors: tumour infiltration in surrounding tissue (T3), tumour invasion into adjacent organs or thrombus in the vena cava or renal vein (T4), positive lymph node (N1), but no distant metastasis (MO) and stage IV, the presence of distant metastases irrespective of tumour size or lymph node status (T1-T4 NO-N1M1).

For determination of serum levels at the time of radiological response, the sample closest to date of response or progression was selected. Serum levels were defined as $<14$ or $\geq 14 \mathrm{mg} /$.

\section{Somatic mutation analysis}

Sections of the stained slides, which contained $>80 \%$ tumour cells, were selected for DNA isolation and mutation analysis. From the selected sections, tissuepunches were taken with a tissue microarrayer (TMA; Beecher, Sun Prairie, WI, USA). DNA was isolated from three tissue cores using the Nucleospin Tissue kit (Marcherey-Nagel, Bethlehem, PA, USA) according to manufacturer's instructions with modifications; tissue cores were deparaffinised once in $1 \mathrm{ml}$ xylene and washed in $100 \%$ ethanol, proteinase-K digestion was performed overnight and DNA was eluted in water. DNA concentrations were measured using Nanodrop 1000 (Isogen, De Meern, The Netherlands).

After DNA isolation, EGFR, BRAF, KRAS and PIK3CA somatic mutation analysis was performed using an allele-specific quantitative real-time PCR (qPCR) with hydrolysis probes (16) for the mutation hotspots: BRAF c.1799T $>$ A (p.V600E), KRAS (c.34G $>$ A, p.G12S/c.34G $>$ C, p.G12R/c.34G $>$ T, p.G12C/c.35G $>$ A, p.G12D/c.35G $>$ C, p.G12A/c. 35G $>$ T and p.G12V/ c.38G $>$ T, p.G13V) and PIK3CA (c.3140 A>G, p.H1047R/c.1624G > A, p.E542K) and EGFR c.2573T $>\mathrm{G}$ (p.L858R) and exon 19 deletions were detected as previously described $(26,27)$. Oligonucleotide and probe sequences are available upon request.

Sanger sequencing of EGFR exons 18-21 and TP53 exons 5-8 was performed using PCR and direct DNA sequencing, in order to find additional mutations. Sanger sequencing was performed at the Leiden Genome Technology Centre using both forward and reverse M13 primers. Data were analysed with Mutation Surveyor DNA analysis software version 3.24 (Softgenetics LLC, State College, PA, USA).

\section{AKT, phosphorylated AKT, ERK, pERK, $\beta$-catenin and PTEN immunohistochemistry}

A tissue microarray was constructed containing three representative tissue cores (of $0.6 \mathrm{~cm}$ in diameter) from each tumour (17). Four-micrometer tissue sections were cut and transferred to glass slides using a tapetransfer system (Instrumedics, Hackensack, NJ, USA). A standard indirect immunohistochemistry (IHC) procedure was performed including citrate antigen retrieval (0.01 M, pH 6.0), blockage of endogenous peroxidase activity and diaminobenzidine development. The following primary antibodies were used: rabbit antiphospho-p44/42 MAPK (Erk1/2) (Thr202/Tyr204) 
(1:50, \#9101, Cell Signalling Technology, Danvers, MA, USA), mouse monoclonal 3A7 anti-p44/42 MAPK (Erk1/2) (1:1000, \#9107), Cell Signalling Technology, rabbit monoclonal (D9E) anti-phospho-Akt (Ser473, 1:25, \#4060), Cell Signalling Technology, mouse monoclonal (40D4) anti-Akt (pan, 1:500, \#2920), monoclonal mouse anti-human PTEN (1:400, 6H2.1, Dako, Glostrup, Denmark) and purified mouse monoclonal anti $\beta$-catenin (1:250, \#610154, BD Transduction Laboratories, Bedford, MA, USA).

AKT, phosphorylated AKT (pAKT), ERK, pERK and PTEN were assessed by the presence or absence of cytoplasmic and/or nuclear staining. $\beta$-Catenin was assessed by the presence of nuclear staining as an indication of activation. Scoring was performed according to prevalence and intensity of cytoplasmic/nuclear staining. Tumours were divided into four groups. Group 1, negative staining $(-)$; group 2 , weak positive staining $(+/-)$; group 3, positive staining $(+)$ and group 4 , strong positive staining $(++)$.

\section{Statistical analysis}

Clinical characteristics of the patients were presented as mean with S.D. or median with range as appropriate. Survival curves were calculated using Kaplan-Meier method. The influence of different clinicopathological parameters on survival was evaluated using MantelCox log-rank test.

Mitotane-related overall survival (MROS) was calculated from the date of mitotane initiation. Only patients with at least 3 months of mitotane monotherapy were included in the MROS analysis. Overall survival (OS) was calculated from the date of diagnosis. Differences in survival according to clinicopathological parameters, mutation status and protein expression were analysed using Cox-regression analysis. Differences in patient and tumour characteristics between responders and non-responders were analysed using Pearson $\chi^{2}$ test and Fisher's exact test. Differences in age, van Slooten index, tumour weight and tumour size were calculated using the Student's $t$-test. A $P$ value $<0.05$ was considered significant. All analyses were performed using SPSS version 15.0.

\section{Results}

\section{Patient characteristics}

Tumour samples of 47 ACCs (30 primary ACCs and 17 recurrences), obtained from a series of 47 patients, were used for IHC and mutation analysis. Patients presented with stage II in $42.6 \%$ of cases $(n=20)$, stage III in $14.9 \%(n=7)$ and stage IV in $42.6 \%(n=20)$. Median OS was 44 months. Mitotane was given as monotherapy for locally advanced or metastatic disease. In $42.2 \%$ of the cases, mitotane was given for primary ACC $(n=19$; patients $1-7,9,11-13,15,20,22-24,36,39$ and 43 ; Table 1$)$, in $51.1 \%(n=16$, patients $16-19,21,25-27$, 29, 31, 33-36, 41, 42 and 47; Table 2) for first recurrence and in $6.67 \%(n=3$, patients 38,40 and 46 ; Table 2) for the second recurrent disease.

\section{Serum levels and response to mitotane}

Of the 47 included patients, 37 patients were available for evaluation of mitotane response. Two patients were excluded from the mitotane response analysis as they did not receive monotherapy but adjuvant mitotane therapy. Another eight patients died before first CT evaluation; therefore, these patients could not be classified as a responder or non-responder.

Objective response was seen in 14 patients, stable disease in four patients and progressive disease in 19 patients. Mitotane serum levels $\geq 14 \mathrm{mg} / \mathrm{l}$ were seen in 13 responders $(92.9 \%)$ and in three $(15.8 \%)$ non-responders. Levels $\geq 14 \mathrm{mg} / \mathrm{l}$ were significantly associated with treatment response $\left(\chi^{2} P=0.00\right)$. MROS of responders was significantly longer than survival observed for non-responding patients (median 51 vs 7 months, $P=0.01$ ), even after correction for age, gender, functionality and stage at diagnosis $(P=0.02) \quad$ (Fig. 1). After correction for mitotane serum levels $<$ or $\geq 14 \mathrm{mg} /$, the survival benefit disappeared $(P=0.71)$. No relationship was found between the presence of hormonal excess and response to mitotane treatment $(P=0.32)$.

\section{Mutation analysis of EGFR, KRAS, BRAF and PIK3CA}

Hotspot mutation analysis of EGFR exon 19 deletions (del 19) and EGFR 21 (c.2573T $>$ G, p.L858R) was

Table 1 TP53 mutations found in 46 adrenocortical carcinoma samples.

\begin{tabular}{|c|c|c|c|}
\hline Patient & Exon & Mutation & \\
\hline 1 & 5 & c. $430 \mathrm{C}>\mathrm{T}, \mathrm{p} . \mathrm{Q} 44 \mathrm{X}$ & Nonsense \\
\hline 9 & & c. $437 G>A, p . W 146 W X$ & Nonsense \\
\hline 11 & & $\begin{array}{l}\text { c. } 452 \mathrm{C}>\mathrm{T}, \text { p.P151PL, } \\
\text { c. } 453 \mathrm{C}>\mathrm{T}, \text { p.P151P }\end{array}$ & Missense \\
\hline 13 & & c.524G > A, p.R175H & Missense \\
\hline 17 & & c.446_447delCC & Deletion \\
\hline 38 & & c. $381 \bar{C}>T, p . S 127 S S$ & Silent \\
\hline 46 & & c. $464 \mathrm{C}>\mathrm{T}$, p.T155L & Silent \\
\hline 4 & 6 & c. $606 \mathrm{~T}>\mathrm{A}$, p.R202RR & Silent \\
\hline 7 & & c.667C > T, p.P223PS & Missense \\
\hline 10 & & c. $578 \mathrm{~A}>\mathrm{T}$, p. $\mathrm{H} 193 \mathrm{~L}$ & Missense \\
\hline 17 & & c.636 del T & Silent \\
\hline 24 & & 628 634delAACACTT & Deletion \\
\hline 37 & & c. $626 \mathrm{G}>\mathrm{A}, \mathrm{p} . \mathrm{R} 209 \mathrm{RK}$ & Missense \\
\hline 17 & 7 & c.746G $>$ A, p.R249RK & Missense \\
\hline 8 & 8 & c. $855 \mathrm{G}>\mathrm{A}$, p.E285EE & Silent \\
\hline 43 & & $\begin{array}{l}\text { c. } 833 C>A,(c .833 C>A), \\
\text { p.P278H }\end{array}$ & Missense \\
\hline
\end{tabular}

NA, not available. 
Table 2 Immunohistochemical results for primary and recurrent ACC.

\begin{tabular}{|c|c|c|c|c|c|c|c|c|c|c|c|c|}
\hline $\begin{array}{l}\text { Pt } \\
n=47\end{array}$ & ACC & $\begin{array}{c}\text { P53 mut } \\
n=46\end{array}$ & $\begin{array}{c}\text { EGFR } \\
n=46\end{array}$ & $\begin{array}{c}\text { PIK3CA } \\
\text { KRAS } \\
n=46\end{array}$ & $\begin{array}{l}\text { BRAF } \\
n=45\end{array}$ & $\begin{array}{c}\text { AKT } \\
n=45\end{array}$ & $\begin{array}{l}\text { pAKT } \\
n=45\end{array}$ & $\begin{array}{c}\text { ERK } \\
n=45\end{array}$ & $\begin{array}{l}\text { pERK } \\
n=45\end{array}$ & $\begin{array}{l}\text { VERK } \\
n=45\end{array}$ & $\begin{array}{c}\boldsymbol{\beta} \text {-Catenin } \\
n=43\end{array}$ & $\begin{array}{c}\text { PTEN loss } \\
n=45\end{array}$ \\
\hline 1 & $\mathrm{Pr}$ & n.m & 0 & $0 / 0$ & 0 & + & + & $+1-$ & $+1-$ & - & + & No \\
\hline 2 & $\mathrm{Pr}$ & 0 & 0 & $0 / 0$ & 0 & + & $+1-$ & + & $+1-$ & - & + & No \\
\hline 3 & $\mathrm{Pr}$ & 0 & 0 & $0 / 0$ & 0 & - & - & $+1-$ & - & - & + & No \\
\hline 4 & $\mathrm{Pr}$ & s.m & NA & NA & NA & NA & NA & NA & NA & NA & NA & NA \\
\hline 5 & $\mathrm{Pr}$ & 0 & s.m & $0 / 0$ & 0 & + & $+1-$ & $+1-$ & - & - & - & No \\
\hline 6 & $\operatorname{Pr}$ & 0 & 0 & $0 / 0$ & 0 & + & $+1-$ & + & $+1-$ & + & + & No \\
\hline 7 & $\mathrm{Pr}$ & $\mathrm{m} \cdot \mathrm{m}$ & 0 & $0 / 0$ & 0 & + & - & + & - & - & + & No \\
\hline 8 & $\mathrm{Pr}$ & s.m & s.m & $0 / 0$ & 0 & + & - & + & $+1-$ & + & + & No \\
\hline 9 & $\mathrm{Pr}$ & n.m & s.m & $0 / 0$ & 0 & $+1-$ & $+1-$ & + & + & - & - & No \\
\hline 10 & $\mathrm{Pr}$ & m.m & 0 & $0 / 0$ & 0 & + & + & + & + & - & + & No \\
\hline 11 & $\mathrm{Pr}$ & $\mathrm{m} \cdot \mathrm{m}$ & 0 & $0 / 0$ & 0 & + & $+1-$ & + & $+1-$ & - & + & No \\
\hline 13 & $\mathrm{Pr}$ & m.m & 0 & $0 / 0$ & 0 & + & $+1-$ & $+1-$ & + & $+1-$ & - & No \\
\hline 14 & $\operatorname{Pr}$ & NA & 0 & $0 / 0$ & 0 & + & - & + & - & $+1-$ & + & No \\
\hline 15 & $\mathrm{Pr}$ & 0 & 0 & $0 / 0$ & 0 & + & + & + & $+1-$ & $+1-$ & NA & No \\
\hline 16 & $\mathrm{Pr}$ & NA & 0 & $0 / 0$ & 0 & + & + & + & + & $+1-$ & NA & No \\
\hline 17 & $\mathrm{Pr}$ & del; m.m & s.m & $0 / 0$ & 0 & + & $+1-$ & + & + & + & + & No \\
\hline 19 & $\mathrm{Pr}$ & NA & 0 & $0 / 0$ & 0 & + & + & + & + & - & + & No \\
\hline 21 & $\mathrm{Pr}$ & NA & 0 & $0 / 0$ & 0 & NA & NA & NA & NA & NA & NA & NA \\
\hline 22 & $\mathrm{Pr}$ & 0 & 0 & $0 / 0$ & 0 & + & $+1-$ & + & $+1-$ & - & - & No \\
\hline 24 & $\operatorname{Pr}$ & del & 0 & $0 / 0$ & 0 & + & $+1-$ & + & - & - & - & No \\
\hline 26 & $\mathrm{Pr}$ & 0 & $\begin{array}{l}\mathrm{m} . \mathrm{m} ; \\
\text { s.m }\end{array}$ & $0 / 0$ & 0 & + & + & + & - & - & - & No \\
\hline 27 & $\mathrm{Pr}$ & 0 & 0 & $0 / 0$ & 0 & + & $+1-$ & + & - & - & - & No \\
\hline 28 & $\mathrm{Pr}$ & 0 & 0 & $0 / 0$ & 0 & + & - & + & - & - & + & No \\
\hline 29 & $\operatorname{Pr}$ & 0 & 0 & $0 / 0$ & 0 & + & - & + & - & - & + & No \\
\hline 31 & $\mathrm{Pr}$ & NA & 0 & $0 / 0$ & 0 & + & $+1-$ & + & $+1-$ & + & - & No \\
\hline 37 & $\mathrm{Pr}$ & $\mathrm{m} \cdot \mathrm{m}$ & 0 & $0 / 0$ & 0 & + & + & - & $+1-$ & - & + & No \\
\hline 38 & $\mathrm{Pr}$ & s.m & 0 & $0 / 0$ & 0 & + & + & + & - & $+1-$ & - & No \\
\hline 41 & $\mathrm{Pr}$ & 0 & 0 & $0 / 0$ & 0 & + & $+1-$ & + & - & $+1-$ & - & No \\
\hline 42 & $\operatorname{Pr}$ & 0 & $\mathrm{~m} \cdot \mathrm{m}$ & $0 / 0$ & + & + & $+1-$ & + & - & - & - & No \\
\hline 44 & $\mathrm{Pr}$ & 0 & 0 & $0 / 0$ & 0 & + & + & - & $+1-$ & $+1-$ & + & No \\
\hline 12 & $\mathrm{R}$ & NA & 0 & $0 / 0$ & 0 & + & $+1-$ & $+1-$ & - & - & - & No \\
\hline 18 & $R$ & 0 & 0 & $0 / 0$ & 0 & + & - & + & $+1-$ & - & - & No \\
\hline 20 & $\mathrm{R}$ & 0 & 0 & $0 / 0$ & 0 & + & + & + & $+1-$ & $+1-$ & + & No \\
\hline 23 & $\mathrm{R}$ & NA & 0 & $0 / 0$ & 0 & + & - & + & - & - & - & No \\
\hline 25 & $\mathrm{R}$ & 0 & 0 & $0 / 0$ & 0 & - & - & + & - & - & + & No \\
\hline 30 & $\mathrm{R}$ & 0 & 0 & $0 / 0$ & 0 & + & - & $+1-$ & $+1-$ & $+1-$ & - & No \\
\hline 32 & $\mathrm{R}$ & 0 & 0 & $0 / 0$ & 0 & + & $+1-$ & + & $+1-$ & - & + & No \\
\hline 33 & $\mathrm{R}$ & NA & 0 & $0 / 0$ & 0 & $+1-$ & - & $+1-$ & $+1-$ & - & - & No \\
\hline 34 & $\mathrm{R}$ & NA & 0 & NA & NA & $+1-$ & - & + & - & - & - & No \\
\hline 35 & $\mathrm{R}$ & 0 & 0 & $0 / 0$ & 0 & + & + & + & - & $+1-$ & - & No \\
\hline 36 & $\mathrm{R}$ & 0 & 0 & $0 / 0$ & 0 & + & + & + & $+1-$ & $+1-$ & + & No \\
\hline 39 & $\mathrm{R}$ & 0 & 0 & $0 / 0$ & 0 & + & $+1-$ & + & $+1-$ & - & + & No \\
\hline 40 & $\mathrm{R}$ & 0 & 0 & $0 / 0$ & 0 & + & + & + & + & $+1-$ & + & No \\
\hline 43 & $\mathrm{R}$ & m.m & s.m & $0 / 0$ & 0 & + & + & + & ++ & $+1-$ & + & No \\
\hline 45 & $\mathrm{R}$ & 0 & 0 & $0 / 0$ & 0 & + & $+1-$ & + & $+1-$ & - & - & No \\
\hline 46 & $\mathrm{R}$ & s.m & m.m & $0 / 0$ & 0 & + & $+1-$ & + & $+1-$ & - & - & No \\
\hline 47 & $\mathrm{R}$ & NA & 0 & $0 / 0$ & 0 & + & $+1-$ & + & - & + & + & No \\
\hline
\end{tabular}

Pt, patient; Pr, primary tumour; R, recurrence; - , negative staining; $(+/-)$, weak positive staining; $(+)$, positive staining; $(++)$, strong positive staining; NA, not available; n.m, non-sense mutation; m.m, missense mutation; s.m, silent mutation; del, deletion; 0 , no mutation.

successfully performed in 45 samples using a hydrolysis probe. No mutations were found. Additional DNA sequencing of EGFR revealed ten mutations, of which seven were silent.

One of the three remaining mutations was found to be a single nucleotide polymorphism (SNP) (exon 20, c. $2271 \mathrm{G}>\mathrm{A}$, p.787A $>$ T, patient 42). Two missense mutations were found in exon 21 (c.2590G $>A$, p.864A $>$ T, patient 26 , c. $2765 \mathrm{G}>\mathrm{GA}$, p.866E $>\mathrm{EK}$, patient 46) (Fig. 2). One activating BRAF mutation (c. 1799T > A, p V600E) was found. No mutations were found in KRAS or PIK3CA.

\section{TP53 mutation analysis}

DNA sequencing of TP53 exons 5-8 could be obtained in the FFPE DNA of 46 ACC samples. In 37 tumours, DNA sequencing of all exons was successful. Sequencing analysis revealed 16 mutations (Table 1). TP53 mutations were more common in patients presenting 


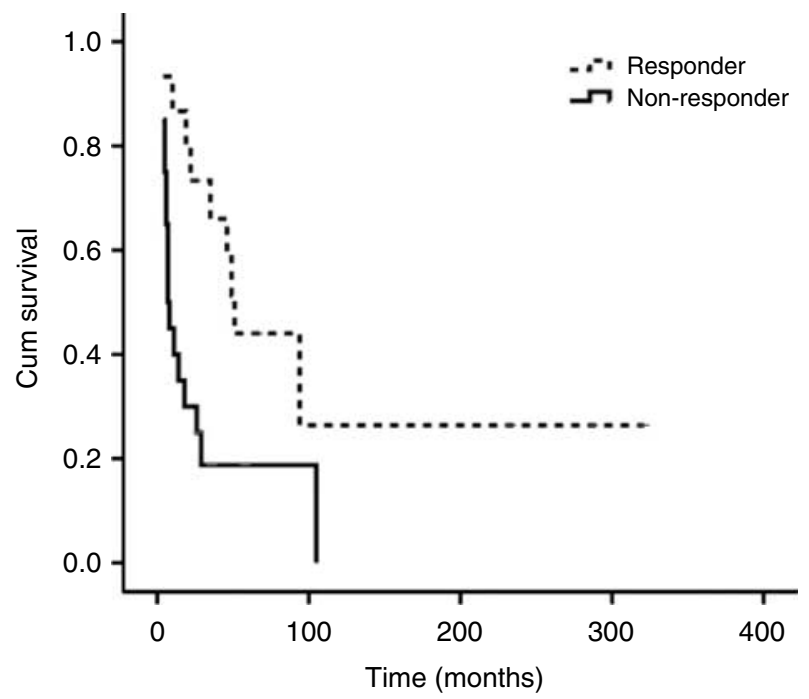

Figure 1 Overall survival from date of mitotane initiation (MROS) for mitotane responders $(n=14)$ and non-responders $(n=19)$. Responders showed a significantly longer OS (median 51 months, range 42-70 months) compared with non-responders (median 7 months, range 5-9 months, $P=0.01$ ).

with stage IV tumours. The median survival of patients with a TP53 mutation was 11 vs 51 months for patients without a TP53 mutation $(P=0.19)$.

\section{PTEN, AKT, phospho-AKT, ERK and phospho-ERK IH}

IHC data were available for 45 tumours (see Table 2 for an overview of the immunohistochemical scoring for each tumour). None of the tumours showed loss of PTEN immunoreactivity. AKT expression was also positive overall. pAKT was detected in 31\% of tumours (Fig. 3). Median OS of 30 months was observed in patients with pAKT-negative tumours, 44 months in patients with pAKT weak positive tumours and 95 months in patients with pAKT-positive tumours $(P=0.163)$. A shorter median survival was found for patients with tumours without pAKT staining compared with patients with pAKT-positive tumours $(+$ and $+/-$ ) $(30$ and 59 months respectively, $P=0.06)$.

ERK staining was positive in the majority of samples $(80 \%)$. In $60 \%$ of the samples, phosphorylation of ERK was observed. Seven of these samples (26\%) showed strongly positive nuclear staining (Fig. 4). Median survival for patients with perk-negative tumours was 73 months, for patients with perk-positive tumours 23 months and for patients with pERK strongly positive tumours 14 months. A trend towards shorter survival was observed for patients with perk-positive tumours $(P=0.08)$ compared with patients with perk-negative tumours. No difference in ERK or pERK staining was observed between primary ACC and recurrences $\left(\chi^{2}, P=0.10, P=0.28\right.$ respectively).
A remarkable positive pERK staining of endothelial cells of blood vessels of 18 ACC samples (40\%) was observed. Five samples even showed a very strong endothelial staining (Fig. 4). No significant survival difference was seen between patients with tumours with negative, positive and strongly positive endothelial staining $(P=0.09)$.

\section{$\beta$-Catenin IH}

$\beta$-Catenin staining results were successfully obtained for 43 samples. Positive nuclear staining was observed in 23 samples $(53.5 \%)$. Median survival of patients with tumours with nuclear immunostaining was 22 vs 59 months for patients with tumours with negative staining $(P=0.30)$. In the group of primary ACCs $(n=30)$, a trend towards shorter survival was revealed (10 vs 59 months, $P=0.06$ ).

\section{Correlation of mutation status, IHC and mitotane response}

No relationship was found between the presence of an EGFR mutation and immunostaining of any of the downstream effectors. Samples harbouring an EGFR mutation showed negative or weak positive staining of pERK and weak positive or positive staining of pAKT. No relationship was found between mutation status, IH results and mitotane response.

\section{Discussion}

We were not able to find a relationship between the EGFR pathway and mitotane response. We did find two
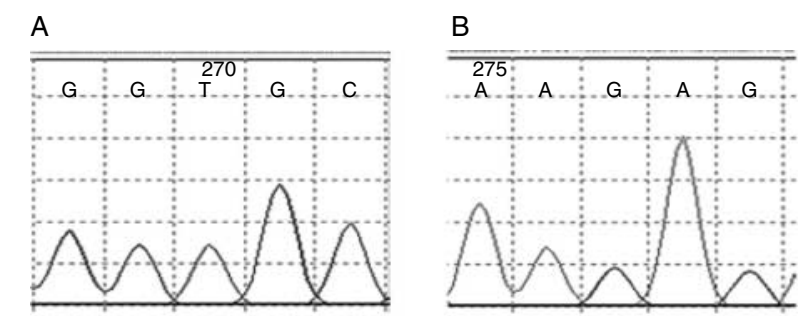

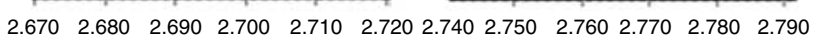
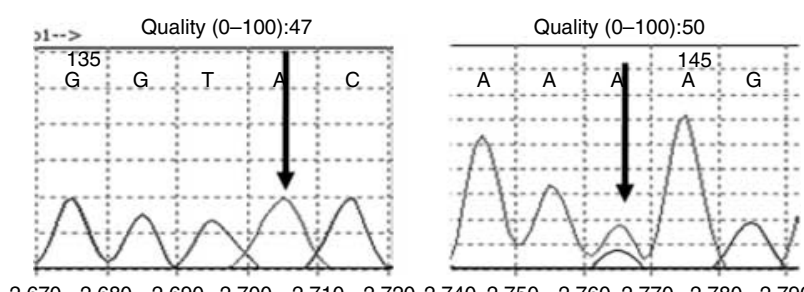

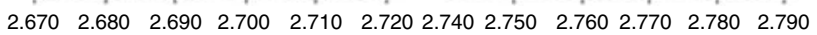
p.A864T

p.E866E/K

Figure 2 EGFR mutation analysis by direct sequencing. The wild-type sequence is shown above, the corresponding mutated sequence is shown below. (A) c.2590GOA, p.A864T. (B) c.2765 GOGA, p.866EOEK. 

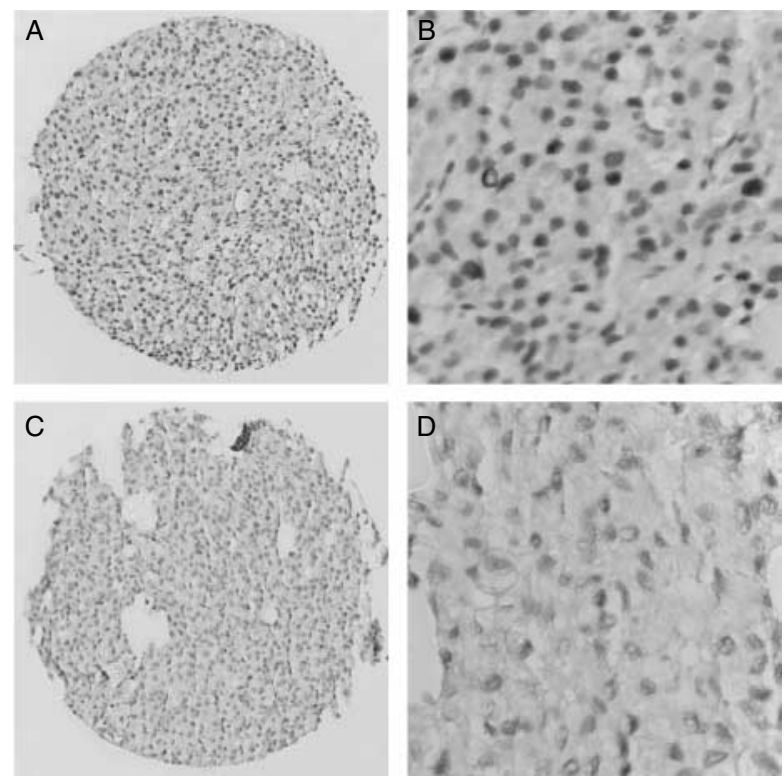

Figure 3 pAKT IH in a TMA of ACC tumours (ACC-TMA). ACC 1 shows positive nuclear staining (A, magnification $\times 125$; $B$, magnification $\times 500)$. ACC 3 shows negative staining for $\mathrm{pAKT}$ $(C$, magnification $\times 125$; $D$, magnification $\times 500)$.

mutations of the EGFR TK domain. The c.2590G $>$ A, p.864A $>$ T (patient 26, Table 2 and Fig. 2) EGFR mutation was described previously in a non-small-cell lung carcinoma and was suggested to respond to EGFR TKI (18). For the other EGFR TK domain mutation (p.866E $>$ K) found in our study, unresponsiveness upon EGFR TKI treatment was described (19).

We also identified BRAF (c. 1799T >A, p.V600E) mutation in one ACC. This patient also had an SNP on EGFR exon 20 (c.2271G >A, p787A > T), probably unresponsive to EGFR TKI treatment. Specific targeting of BRAF, however, may be an option in this patient as BRAF targeting has recently been shown to be effective in melanomas (20).

Until now, only four mutations of the EGFR TK domain have been reported in $\operatorname{ACC}(21,22)$. Kotoula et al. described four activating EGFR mutations, three missense (c.2168T >C, exon 18, c.2354C >A, exon 20, and c.2533G $>$ A, exon 21) and one non-sense mutation (c.2193G $>$ A, exon 19) in 35 ACCs. The observed mutations were also described earlier in lung and colon carcinoma, but the response to EGFR-TKI therapy was not reported $(23,24,25)$. Recently, Adam et al. (22) found no mutations in 30 ACCs.

The expected incidence of the EGFR mutation in ACC is rather low, $\sim 5 \%$ of patients. The role of the EGFR TKIs is therefore expected to be minor. Furthermore, the majority of patients harbouring an EGFR mutation will be treated with mitotane, which is known to interact with EGFR-TKIs (26).

A second result of potential clinical relevance is that in the remaining ACCs without activating EGFR mutations, no downstream mutations were present in KRAS, BRAF or PIK3CA and preserved PTEN expression was seen.

MABs like cetuximab and panitumumab have been used in colorectal carcinoma without mutations of EGFR or downstream effectors. Patients are selected for MABs based on EGFR protein expression on the membrane of the tumour (27). Since the majority of ACCs have neither EGFR nor downstream mutations of KRAS, BRAF or PIK3CA, MABs could be of interest, especially since EGFR protein expression is observed in the majority of ACC (22). However, before the initiation of a clinical trial, the EGFR gene status should be determined as EGFR protein expression does not correlate with response to the EGFR MABs $(28,29)$.

The presence of BRAF and KRAS mutations in ACC was reported in the literature to be present in only $1.6 \%$ $(1 / 59)$ and $6.7 \%(1 / 15)$ of ACC respectively $(21,30)$. The presence of oncogenic PIK $3 C A$ mutations in ACC has not previously been studied.

From this study, we concluded that the mutation status of EGFR, KRAS, BRAF, PIK3CA and TP53 did not correlate with mitotane response. The same holds true for AKT, pAKT, ERK, pERK and $\beta$-catenin expression.

In addition to the relationship of EGFR and downstream pathways with mitotane response, we also studied the relationship with OS. Phosphorylation of pERK and pAKT and downregulation (loss) of PTEN have been associated with disease progression as well as shorter disease-free and OS in multiple malignancies.
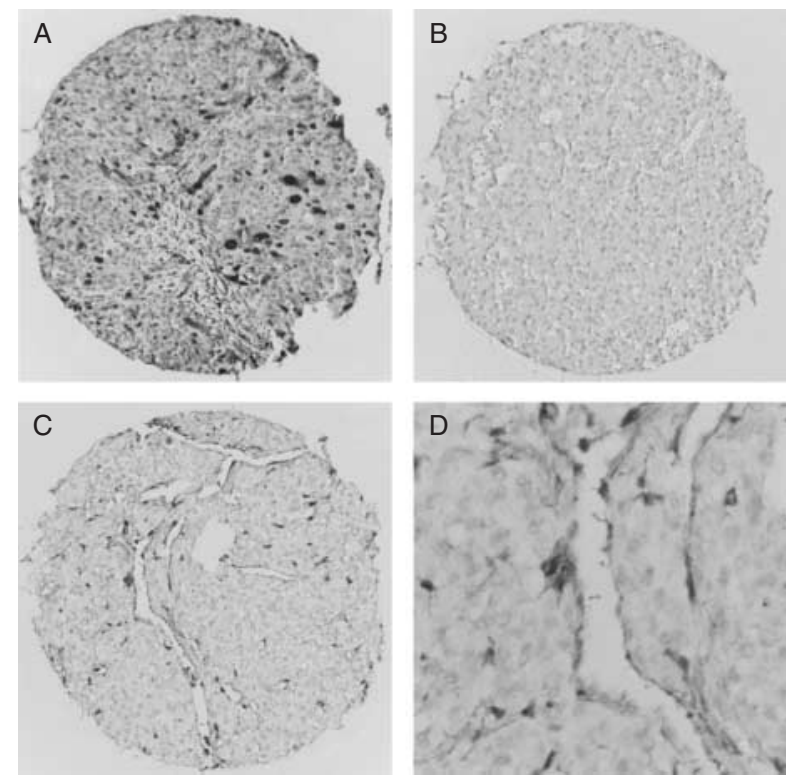

Figure 4 PERK IH of ACC-TMA. ACC 17 (A) shows strong nuclear staining in a fraction of ACC cells. Furthermore, endothelial cell positivity can be observed. In ACC 7 (B), no positivity of ACC or endothelial cells is seen. In ACC 35 (C, magnification $\times 125$; $D$, magnification $\times 500$ ), only positive nuclear and cytoplasmic staining of endothelial cells is observed. 
The role of these molecules in ACC has not yet been established.

Overexpression of ERK, as well as aberrant phosphorylation of ERK, has been associated with poor prognosis in several tumours (31). We were the first to study the expression of both markers in ACC and we found a trend towards shorter survival for pERKpositive samples. However, this survival benefit was not found to be significant, probably because of the small sample size. Furthermore, we found a remarkable pERK staining in endothelial cells of blood vessels of unknown biological significance in 18 ACCs.

Another remarkable finding was the observation of a trend towards shorter survival in pAKT-negative samples $(P=0.06)$. This is a rather unexpected finding as pAKT positivity has been associated with shorter survival in other malignancies (32). As pAKT and pERK have not yet been extensively studied in ACC, future studies are needed in order to demonstrate involvement and relevance of this pathway in ACC.

$\beta$-Catenin and TP53 are frequently mutated in ACC. Both have been suggested as markers of malignancy $(33,34)$. Furthermore, nuclear $\beta$-catenin staining has recently been shown to be associated with a poor prognosis. In our study, we were not able to confirm these findings as only a trend towards shorter survival was observed in primary ACCs with positive nuclear $\beta$-catenin. Furthermore, no relationship was found between the presence of a TP53 mutation and survival. However, due to the nature of the FFPE DNA, some mutations might have been missed in the current study.

Mitotane remains one of the most effective treatments for ACC; however, limitations do exist so that progress in the development and understanding of mechanism of action of this drug is highly important. In order to improve prediction of tumour response to mitotane treatment, we determined the mutation status of EGFR and downstream signalling pathways. Although we were not able to demonstrate a relationship between the EGFR pathway and mitotane response, we were able to confirm the relevance of the therapeutic threshold of $14 \mathrm{mg} / \mathrm{l}$ as serum levels $\geq 14 \mathrm{mg} / \mathrm{l}$ were significantly associated with objective response. Furthermore, significantly longer MROS was seen in responding patients. The observation of disappearance of the survival benefit after correction for serum level even strengthens the importance of the therapeutic threshold. As mitotane is the most active treatment available, further progress regarding predictors of response is still needed. Although our study has limitations, such as the use of FFPE material, the use of primary as well as recurrent ACC, we feel that it contributes to the improvement of insight into the pathogenesis and the behaviour of ACC.

In conclusion, no relationship was found between the EGFR pathway and mitotane response. Our data suggest that the role of EGFR TKIs in ACC is limited. Treatment with EGFR MABs on the other hand might be beneficial for a larger group of patients. The possible efficacy of this therapy in ACC should be evaluated in future trials. Furthermore, our data confirm the importance of the $14 \mathrm{mg} / \mathrm{l}$ cut-off level of $o, p^{\prime}$-DDD in the therapeutic management of ACC patients as improved survival was demonstrated for patients with serum levels $\geq 14 \mathrm{mg} / \mathrm{l}$ independently of the occurrence of response.

\section{Declaration of interest}

The authors declare that there is no conflict of interest that could be perceived as prejudicing the impartiality of the research reported.

\section{Funding}

This research did not receive any specific grant from any funding agency in the public, commercial or not-for-profit sector.

\section{Author contribution statement}

I G C Hermsen prepared the manuscript, collected the patient data, prepared the TMA, carried out the DNA isolation and mutational analysis and drafted the manuscript. H R Haak participated in the design of the study and drafting of the manuscript. T M A Kerkhofs helped collect patient data in SPSS file and helped perform the statistical analysis. R R de Krijger, W W de Herder, H W Wilmink, J WA Smit and $\mathrm{H}$ J Gelderblom contributed to acquisition of patient data. N F $\mathrm{C} C$ de Miranda carried out and interpreted the immunoassays. $\mathrm{R}$ van Eijk participated in the sequence alignment (Taqman). $T$ van Wezel $R$ van Eijk participated in the sequence alignment and interpretation of somatic mutational analysis. JM was involved in design, drafting and revising the manuscript critically. All authors read and approved the final manuscript.

\section{References}

1 Hahner S \& Fassnacht M. Mitotane for adrenocortical carcinoma treatment. Current Opinion in Investigational Drugs 20056 386-394.

2 Gonzalez RJ, Tamm EP, Ng C, Phan AT, Vassilopoulou-Sellin R, Perrier ND, Evans DB \& Lee JE. Response to mitotane predicts outcome in patients with recurrent adrenal cortical carcinoma. Surgery 2007 142 867-875. (doi:10.1016/j.surg.2007.09.006)

3 Haak HR, Hermans J, van de Velde CJ, Lentjes EG, Goslings BM, Fleuren GJ \& Krans HM. Optimal treatment of adrenocortical carcinoma with mitotane: results in a consecutive series of 96 patients. British Journal of Cancer 199469 947-951. (doi:10. 1038/bjc.1994.183)

4 Baudin E, Pellegriti G, Bonnay M, Penfornis A, Laplanche A, Vassal G \& Schlumberger M. Impact of monitoring plasma 1,1dichlorodiphenildichloroethane (o,p'DDD) levels on the treatment of patients with adrenocortical carcinoma. Cancer 200192 13851392. (doi:10.1002/1097-0142(20010915)92:6<1385::AIDCNCR1461>3.0.CO;2-2)

5 Venkatesh S, Hickey RC, Sellin RV, Fernandez JF \& Samaan NA. Adrenal cortical carcinoma. Cancer 198964 765-769. (doi:10. 1002/1097-0142(19890801)64:3 < 765::AID-CNCR28206403 $33>3.0 . \mathrm{CO} ; 2-\mathrm{I})$

6 Giordano TJ, Kuick R, Else T, Gauger PG, Vinco M, Bauersfeld J, Sanders D, Thomas DG, Doherty G \& Hammer G. Molecular classification and prognostication of adrenocortical tumors by transcriptome profiling. Clinical Cancer Research $2009 \mathbf{1 5}$ 668-676. (doi:10.1158/1078-0432.CCR-08-1067)

7 de Reyniès A, Assié G, Rickman DS, Tissier F, Groussin L, RenéCorail F, Dousset B, Bertagna X, Clauser E \& Bertherat J. Gene expression profiling reveals a new classification of adrenocortical 
tumors and identifies molecular predictors of malignancy and survival. Journal of Clinical Oncology 200927 1108-1115. (doi:10.1200/JCO.2008.18.5678)

8 Ragazzon B, Libé R, Gaujoux S, Assié G, Fratticci A, Launay P, Clauser E, Bertagna X, Tissier F, de Reyniès A et al. Transcriptome analysis reveals that $\mathrm{p} 53$ and $\beta$-catenin alterations occur in a group of aggressive adrenocortical cancers. Cancer Research 2010 70 8276-8281. (doi:10.1158/0008-5472.CAN-10-2014)

9 Kirschner LS. The next generation of therapies for adrenocortical cancers. Trends in Endocrinology and Metabolism 201223 343-350. (doi:10.1016/j.tem.2012.04.001)

10 Gerber DE. Targeted therapies: a new generation of cancer treatments. American Family Physician 200877 311-319.

11 Salomon DS, Brandt R, Ciardiello F \& Normanno N. Epidermal growth factor-related peptides and their receptors in human malignancies. Critical Review in Oncology and Hematology 199519 183-232. (doi:10.1016/1040-8428(94)00144-I)

12 van Slooten H, Schaberg A, Smeenk D \& Moolenaar AJ. Morphologic characteristics of benign and malignant adrenocortical tumors. Cancer 198555 766-773. (doi:10.1002/10970142(19850215)55:4<766::AID-CNCR2820550414>3.0.CO;2-7)

13 Weiss LM. Comparative histologic study of 43 metastasizing and nonmetastasizing adrenocortical tumors. American Journal of Surgical Pathology 19848 163-169. (doi:10.1097/00000478198403000-00001)

14 Therasse P, Arbuck SG, Eisenhauer EA, Wanders J, Kaplan RS, Rubinstein L, Verweij J, Van Glabbeke M, van Oosterom AT, Christian MC et al. New guidelines to evaluate the response to treatment in solid tumors. European Organization for Research and Treatment of Cancer, National Cancer Institute of the United States, National Cancer Institute of Canada. Journal of the National Cancer Institute 200092 205-216. (doi:10.1093/jnci/92.3.205)

15 Fassnacht M, Johanssen S, Quinkler M, Bucsky P, Willenberg HS, Beuschlein F, Terzolo M, Mueller HH, Hahner S \& Allolio B. Limited prognostic value of the 2004 International Union Against Cancer staging classification for adrenocortical carcinoma: proposal for a Revised TNM Classification. Cancer 2009115 243-250. (doi:10.1002/cncr.24030)

16 van Eijk R, Licht J, Schrumpf M, Talebian Yazdi M, Ruano D, Forte GI, Nederlof PM, Veselic M, Rabe KF, Annema JT et al. Rapid KRAS, EGFR, BRAF and PIK3CA mutation analysis of fine needle aspirates from non-small-cell lung cancer using allele-specific qPCR. PLoS ONE 20116 e17791. (doi:10.1371/journal.pone. $0017791)$

17 Hendriks Y, Franken P, Dierssen JW, De Leeuw W, Wijnen J, Dreef E, Tops C, Breuning M, Bröcker-Vriends A, Vasen H et al. Conventional and tissue microarray immunohistochemical expression analysis of mismatch repair in hereditary colorectal tumors. American Journal of Pathology 2003162 469-477. (doi:10.1016/ S0002-9440(10)63841-2)

18 Tsao MS, Sakurada A, Cutz JC, Zhu CQ, Kamel-Reid S, Squire J, Lorimer I, Zhang T, Liu N, Daneshmand M et al. Erlotinib in lung cancer - molecular and clinical predictors of outcome. New England Journal of Medicine 2005353 133-144. (doi:10.1056/ NEJMoa050736)

19 Kancha RK, von Bubnoff N, Peschel C \& Duyster J. Functional analysis of epidermal growth factor receptor (EGFR) mutations and potential implications for EGFR targeted therapy. Clinical Cancer Research 200915 460-467. (doi:10.1158/1078-0432. CCR-08-1757)

20 Sosman JA, Kim KB, Schuchter L, Gonzalez R, Pavlick AC, Weber JS, McArthur GA, Hutson TE, Moschos SJ, Flaherty KT et al. Survival in BRAF V600-mutant advanced melanoma treated with vemurafenib. New England Journal of Medicine 2012366707 714. (doi:10.1056/NEJMoa1112302)

21 Kotoula V, Sozopoulos E, Litsiou H, Fanourakis G, Koletsa T, Voutsinas G, Tseleni-Balafouta S, Mitsiades CS, Wellmann A \& Mitsiades N. Mutational analysis of the BRAF, RAS and EGFR genes in human adrenocortical carcinomas. Endocrine-Related Cancer 200916 565-572. (doi:10.1677/ERC-08-0101)
22 Adam P, Hahner S, Hartmann M, Heinrich B, Quinkler M, Willenberg HS, Saeger W, Sbiera S, Schmull S, Voelker HU et al. Epidermal growth factor receptor in adrenocortical tumors: analysis of gene sequence, protein expression and correlation with clinical outcome. Modern Pathology 201023 1596-1604. (doi:10.1038/modpathol.2010.153)

23 Ichihara S, Toyooka S, Fujiwara Y, Hotta K, Shigematsu H, Tokumo M, Soh J, Asano H, Ichimura K, Aoe K et al. The impact of epidermal growth factor receptor gene status on gefitinib-treated Japanese patients with non-small-cell lung cancer. International Journal of Cancer 2007120 1239-1247. (doi:10.1002/ijc.22513)

24 Tam IY, Chung LP, Suen WS, Wang E, Wong MC, Ho KK, Lam WK, Chiu SW, Girard L, Minna JD et al. Distinct epidermal growth factor receptor and KRAS mutation patterns in non-small cell lung cancer patients with different tobacco exposure and clinicopathologic features. Clinical Cancer Research 200612 1647-1653. (doi:10.1158/1078-0432.CCR-05-1981)

25 Grothey A. EGFR antibodies in colorectal cancer: where do they belong? Journal of Clinical Oncology 201028 4668-4670. (doi:10.1200/JCO.2010.29.3407)

26 Kroiss M, Quinkler M, Lutz WK, Allolio B \& Fassnacht M. Drug interactions with mitotane by induction of CYP3A4 metabolism in the clinical management of adrenocortical carcinoma. Clinical Endocrinology 201175 585-591. (doi:10.1111/j.1365-2265. 2011.04214.x)

27 Argiles G, Dienstmann R, Elez E \& Tabernero J. Panitumumab: a summary of clinical development in colorectal cancer and future directions. Future Oncology 20128 373-389. (doi:10.2217/fon. 12.20)

28 Vallböhmer D, Zhang W, Gordon M, Yang DY, Yun J, Press OA, Rhodes KE, Sherrod AE, Iqbal S, Danenberg KD et al. Molecular determinants of cetuximab efficacy. Journal of Clinical Oncology 200523 3536-3544. (doi:10.1200/JCO.2005.09.100)

29 Saltz LB, Meropol NJ, Loehrer PJS, Needle MN, Kopit J \& Mayer RJ. Phase II trial of cetuximab in patients with refractory colorectal cancer that expresses the epidermal growth factor receptor. Journal of Clinical Oncology 200422 1201-1208. (doi:10.1200/ JCO.2004.10.182)

30 Masi G, Lavezzo E, Iacobone M, Favia G, Palu G \& Barzon L. Investigation of BRAF and CTNNB1 activating mutations in adrenocortical tumors. Journal of Endocrinological Investigation 200932 597-600.

31 Tanaka Y, Terai Y, Tanabe A, Sasaki H, Sekijima T, Fujiwara S, Yamashita Y, Kanemura M, Ueda M, Sugita M et al. Prognostic effect of epidermal growth factor receptor gene mutations and the aberrant phosphorylation of Akt and ERK in ovarian cancer. Cancer Biology \& Therapy 201111 50-57. (doi:10.4161/cbt.11.1. 13877)

32 Fresno Vara JA, Casado E, de Castro J, Cejas P, Belda-Iniesta C \& Gonzalez-Baron M. PI3K/Akt signalling pathway and cancer. Cancer Treatment Reviews 200430 193-204. (doi:10.1016/j.ctrv. 2003.07.007)

33 Reincke M, Karl M, Travis WH, Mastorakos G, Allolio B, Linehan HM \& Chrousos GP. p53 mutations in human adrenocortical neoplasms: immunohistochemical and molecular studies. Journal of Clinical Endocrinology and Metabolism $1994 \mathbf{7 8}$ 790-794. (doi:10.1210/jc.78.3.790)

34 Gaujoux S, Grabar S, Fassnacht M, Ragazzon B, Launay P, Libé R, Chokri I, Audebourg A, Royer B, Sbiera S et al. $\beta$-Catenin activation is associated with specific clinical and pathologic characteristics and a poor outcome in adrenocortical carcinoma. Clinical Cancer Research 201117 328-336. (doi:10.1158/10780432.CCR-10-2006)

Received 30 January 2013

Revised version received 30 March 2013

Accepted 12 April 2013 